\title{
Impact of semi-annual ionospheric total electron content variation on station displacements using single-frequency PPP
}

\author{
Tzu-Pang Tseng ${ }^{1,2}$, C. K. Shum ${ }^{3,4}$, Yu-Shen Hsiao ${ }^{5, *}$, Chung-Yen Kuo ${ }^{6}$, and Wen-Hao Yeh ${ }^{7}$ \\ ${ }^{1}$ Geoscience Australia, Symonston ACT, Australia \\ ${ }^{2}$ Department of Civil Engineering, National Kaohsiung University of Science and Technology, Kaohsiung City, Taiwan \\ ${ }^{3}$ Division of Geodetic Science, School of Earth Sciences, Ohio State University, Columbus, Ohio, USA \\ ${ }^{4}$ Innovation Academy for Precision Measurement Science and Technology, Chinese Academy of Sciences, Wuhan, China \\ ${ }^{5}$ Department of Soil and Water Conservation, National Chung Hsing University, Taichung City, Taiwan \\ ${ }^{6}$ Department of Geomatics, National Cheng Kung University, Tainan City, Taiwan \\ ${ }^{7}$ National Space Organization, Hsinchu City, Taiwan
}

Article history:

Received 17 February 2021

Revised 12 July 2021

Accepted 31 August 2021

Keywords:

Precise point positioning, Single frequency, Station displacement, Ionosphere, Total electron content

Citation:

Tseng, T.-P., C. K. Shum, Y.-S. Hsiao, C.-Y. Kuo, and W.-H. Yeh, 2021: Impact of semi-annual ionospheric total electron content variation on station displacements using singlefrequency PPP. Terr. Atmos. Ocean Sci., 32, 541-551, doi: 10.3319/ TAO.2021.08.31.01

\begin{abstract}
Global Positioning System (GPS) station displacements in this work are derived using the so-called precise point positioning (PPP) technique with low-cost singlefrequency (SF) receivers. In the SF PPP, the ionosphere delay is the largest error source if the satellite orbits and clocks are well modeled. We use two strategies to minimize the ionosphere delay for an internal comparison: (1) correction using the global ionosphere map (GIM), and (2) estimates of the ionospheric total electron content (TEC) from SF observables (SFO). The trends of the station displacements derived from these two strategies consistently present a systematic movement toward the southwest. Here the trend is referred to the slope of a linear function used to fit the displacement data. Such a systematic movement is mainly caused by the semi-annual variation of the ionospheric TEC rather than the seasonal geophysical effect and the high-order ionosphere effect, both of which only cause the station displacements ranging from a few $\mathrm{mm}$ to a few $\mathrm{cm}$. We present a statistical analysis in terms of correlation coefficients between the semi-annual TEC variation and the station displacement. The maximum correlation coefficient is higher than 0.8 in the $\mathrm{U}$ component, followed by the $\mathrm{E}$ and $\mathrm{N}$ components. In addition, the impact of the semi-annual TEC variation on the station displacement is approximately $0.71,0.45$, and $0.92 \mathrm{~m}$ in the north $(\mathrm{N})$, east $(\mathrm{E})$, and height $(\mathrm{U})$ for a region close to the latitude $23^{\circ} \mathrm{N}$ and longitude $121^{\circ} \mathrm{E}$. This suggests that the semi-annual TEC variation should be considered in a time series of station displacements derived by the SF-PPP.
\end{abstract}

\section{INTRODUCTION}

Global Positioning System (GPS) positioning technique has been widely applied to monitor the geodynamics and geological processes, such as landslide monitoring and tectonics (Blewitt and Lavallee 2002; Meier et al. 2010; Hastaoglu and Sanli 2011; Wang 2012). To monitor both the landslide and tectonic motion, the determination of station displacements using GPS is the most direct and efficient means.

If the station displacements as a function of time are known, it is possible to determine the variation of the station

\footnotetext{
* Corresponding author

E-mail:yshsiao@nchu.edu.tw
}

position in terms of the direction and the magnitude, namely the displacement trend. Here, the accuracy of station displacement is dominated by the quality of both the satellite orbit and clock if the precise point positioning (PPP) technique is used (Zumberge et al. 1997; Tseng et al. 2018). In general, the displacement trend is mainly applied to studies of the surface deformation monitoring, e.g., tectonic motion or landslides. The determination of the displacement trend is mainly biased by the seasonal effect, which is the combination of the continental hydrologic, atmospheric (including the tropospheric wet component) and oceanic effects (Jin et al. 2007; Tseng et al. 2017). On the other hand, the high-order ionosphere signal delay can also affect the 
station displacement, which moves toward the south due to the interaction between the earth's magnetic field and the GPS signal propagation direction (Kedar et al. 2003; Hernández-Pajares et al. 2007). For example, the highorder ionosphere delay results in the station displacement southward in the horizontal component and downward in the vertical component over Brazil (Hadas et al. 2017). The above seasonal and high-order ionosphere effects are only detected by a station equipped with a high precision GPS dual-frequency receiver for geodetic surveying purposes. However, a dual-frequency receiver is much more expensive than a single-frequency $(\mathrm{SF})$ receiver.

The objective of this study is to assess the impact of the semi-annual TEC variation on station displacements derived by the SF PPP technique that is obtained in Bernese software (Dach et al. 2015). Experimental areas are selected in Zulin and Chiamoo, which are located in southern Taiwan, near latitude $23^{\circ} \mathrm{N}$ and longitude $121^{\circ} \mathrm{E}$. Two strategies are used to mitigate the ionosphere delays in the SF PPP and compared for their effectiveness: (1) the ionospheric corrections using the Global Ionosphere Model (GIM), and (2) the estimation of ionospheric TEC from SF observables (SFO). Station displacements resulted from these two strategies are used to derive the trend of the station displacements and subsequently, the impact of the semi-annual TEC variation on the station displacement is assessed. The summary and conclusions of this work then follow.

\section{DATA PROCESSING OF SINGLE-FREQUENCY MEASUREMENTS}

In this work, we use GPS SF receivers to collect both the GPS L1 carrier phase and P1 code measurements. The equation of the GPS SF observation can be expressed as follows (Hofmann-Wellenhof et al. 2001):

$$
\begin{aligned}
& \begin{array}{r}
P 1=\rho+c\left(\delta t^{S A T}-\delta t^{R E C}\right)+\left(I_{1}+I_{2}\right)+T+\delta_{c d}+\varepsilon_{P 1} \\
L 1=\rho+c\left(\delta t^{S A T}-\delta t^{R E C}\right)-\left(I_{1}+\frac{1}{2} I_{2}\right) \\
\quad+T+\lambda_{1} N_{1}+\delta_{p h}+\varepsilon_{L 1}
\end{array} \\
& \text { with } I_{1}=\frac{40.3}{f^{2}} \int_{R E C}^{S A T} n d L
\end{aligned}
$$

where $\rho$ denotes the geometric distance between a GPS satellite and a receiver, $f$ is the L1 frequency, $\delta t^{S A T}$ and $\delta t^{R E C}$ are the satellite clock error and the receiver clock error, respectively, $T$ denotes the troposphere delay, $\lambda_{1}$ and $N_{1}$ denotes the L1 wavelength and ambiguity, respectively, $\varepsilon_{P 1}$ and $\varepsilon_{L 1}$ are the code noise and phase noise, respectively, including the multipath effect. $\delta_{p h}$ is the phase hardware delay bias, and $\delta_{c d}$ is the code hardware delay bias for both the satellite and the receiver, $\int_{R E C}^{S A T} n d L$ (in units of electrons per $\mathrm{m}^{2}$ ) is the so-called slant TEC along the GPS signal propagation path $L$ and $n$ denotes the ionosphere electron density, $B_{0}$ denotes the earth's magnetic field and $\theta$ denotes the angle between the GPS signal propagation direction and the $B_{0}$ direction.

The hardware biases are difficult to be separated in the undifferenced form (e.g., PPP) and are highly correlated with clock errors. The code hardware bias at the satellite end is different from satellite to satellite. However, this is not the case for the receiver end. The code bias at the receiver end is a common offset for all pairs of the satellite-receiver observations and thus can be absorbed by estimating the receiver clock in the positioning procedure. However, the code bias at the satellite end needs to be taken into account. In addition, the satellite phase bias is not considered in this work and is mainly used to improve the time convergence of solution due to an effective assistance to resolve the integer phase ambiguity. The troposphere delay consists of a hydrostatic delay and a wet delay, where the hydrostatic delay can be corrected by a tropospheric model (Boehm et al. 2006). The wet delay is usually estimated in the data processing step. The sum of the $\left(I_{1}+I_{2}\right)$ term is the ionosphere delay, which is frequency-dependent, including the first-order $\left(I_{1}\right)$ and second-order $\left(I_{2}\right)$ effects.

In this study, daily TEC variations are approximately dealt with either via the correction using the GIM, or via the SFO to account for the ionosphere delays. The GIM model is obtained from the Center for Orbit Determination in Europe (CODE) and provides the vertical TEC variations, which is then converted into the slant TEC through a mapping function. Furthermore, Liu et al. (2018) reported that the CODE's GIM model agreed with the IGS product to 1.4 TECU. Here, the error caused by the mapping function is ignored due to the fact that the distance between the satellite and the station is much larger than the orbit error and positioning error. In comparison, both the L1 and P1 measurements with different weights are used to estimate the slant TEC in the SFO case (Dach et al. 2015). In this case, the SF TEC is estimated by taking partial derivatives of $\mathrm{L} 1$ and $\mathrm{P} 1$ with respect to TEC in Eq. (1), just like taking partial derivatives of $\mathrm{L} 1$ and $\mathrm{P} 1$ with respect to the station coordinates and the receiver clock. We did not use the linear combination of Eqs. (1) and (2) for positioning. This is because the code noise is 1000 times nosier than the phase noise and thus, the positioning solution may be contaminated by the code noise if the linear combination of L1 and P1 is used. Here, the differential code bias (P1-C1 DCB) is used to account for the $\delta_{c d}$ term in Eq. (1) and to de-correlate with the TEC estimation in the SF PPP.

As a final remark, initial values, including station coordinates, receiver clock and zenith troposphere delay for the SF PPP is obtained by using the code measurements if an external ionosphere model (GIM) is used here. 
Subsequently, the phase measurement is cleaned using the initial coordinates derived by the code measurement, in terms of the cycle slip and outlier detections. With the clean phase measurements, the corrections of the all parameters to the initial values are estimated. The linear combination of L1-P1 may be used for the SF PPP. Although the L1-P1 linear combination can remove the ionosphere effect, such a L1-P1 combination also increases the measurement noise due to the code noise. However, this is not the case for this work. The parameter corrections to the initial values are estimated by the phase measurement.

\section{STATION DISPLACEMENT AND ITS TREND IN GIM AND SFO CASES}

We select Zulin in Kaohsiung City and Chiamoo in Pingtung County, southern Taiwan, as experimental areas for the analysis of station displacement derived by the SF PPP technique. Figure 1 shows the locations of the experimental areas. The data time span used in this work is from DOY 1 to 217, 2015 (1 January 2015 to 1 August 2015).

Daily station displacements are obtained by comparing all positioning solutions with a reference coordinate which is the first-day positioning solution. Figure 2 shows the histogram of the displacements in both the GIM and SFO cases. The station displacements derived by L1 measurements present a normal distribution and the related statistic information is summarized in Table 1 . The standard deviation (STD) in the horizontal component from the SFO case is better than that from the GIM. However, the STD in the vertical component from the SFO case is relatively degraded as compared to that from the GIM case. This is interpreted that the slant TEC is highly correlated with the station-related parameters because the TEC parameter is lumped together with the station coordinates, station receiver clock and station troposphere delay in the estimation procedure.

As an internal comparison, the SFO-derived displacements in the horizontal component are more concentrative than the GIM-derived ones. However, this is not the case for the vertical component that the SFO-derived displacements are relatively discrete as compared to the GIM-derived ones. This is because the GIM model results from the geometry-free observations that remove the orbit error, clock errors and troposphere error. These terms are highly correlated with the accuracy of the vertical component. In comparison, the SFO solution is only originated from the L1 measurement that contains the above errors and all of the errors need to be carefully considered in estimating the slant TEC. Inevitably, these terms are highly correlated with the station-vertical accuracy and are hardly separated without constraints in the estimation process. As such, the SFOderived displacements in the vertical component are more discrete than the GIM-derived ones.

Overall, the displacements range from -2 to $2 \mathrm{~m}$ and such an error range is mainly caused by the combination of the multipath, station- and satellite-related hardware errors and the ionosphere mis-modeling. For example, the GIM results from an assumption that the ionosphere is concentrated in a thin shell at an altitude of $400-450 \mathrm{~km}$ above the earth's surface. Such an assumption is not physically true because the ionosphere can be divided into several layers along the electron density profile (Gao and Liu 2002).

On the other hand, for the determination of displacement trend, the absolute accuracy of the station position is not concerned but the relative displacement with respect to the reference solution is crucial. Here, the trend is referred to the slope of a linear function used to fit the displacement data. The trend of the station displacement is obtained by comparing the daily SF PPP coordinate solution to the one on the first day. As such, the mean bias in Table 1 can be safely ignored in the analysis of the displacement trend. In order to validate our work, the SF-derived displacement trend is then compared to that derived by the double difference solution. Figures 3 and 4 show the displacement trends derived by GIM and SFO over Zulin and Chiamoo. The trends given by the relative positioning are regarded as the ground truth and are provided by the Central Geological Survey (CGS) of Taiwan. Reference stations close to Chiamoo and Zulin are used to create double-differenced observations. The baselines between the reference stations and those monitoring stations are less than $10 \mathrm{~km}$ in order to remove the common ionospheric effect. The GIM-derived trends in the horizontal component move toward the southwest similar to the SFO-derived ones. However, this is not the case for the solution resulted from the relative positioning, whose horizontal displacements move toward the northwest and do not present the systematic movement. Additionally, the vertical displacements from both the GIM and SFO cases are mostly negative. In the following, the systematic movement in the SF PPP is assessed.

\section{CORRELATION BETWEEN SEMI-ANNUAL TEC VARIATIONS AND STATION DISPLACEMENTS}

According to Tseng et al. (2017), the seasonal effect, which is mostly related to the mass redistribution of the earth, on station displacement is only at mm level and can be safely ignored in this work. Additionally, the positioning solution heavily relies on the phase measurement, so the high-order ionosphere $I_{2}$ term may also bias the displacement trend due to the interaction between the geomagnetic field and the GPS signal propagation direction (Kedar et al. 2003; Hernández-Pajares et al. 2007).

According to the $I_{2}$ formula of Eq. (2), the sign of the $I_{2}$ effect is dominated by the $B_{0} \cos \theta$ term. Figure 5 shows a simulation of $B_{0} \cos \theta$, where $B_{0}$ is resulted from a dipole geomagnetic model. Here, the station is assumed to be located at a latitude of $22.5^{\circ}$ approximately near the 
experiment area of Fig. 1. The phase delay or phase advance is mainly associated with the interaction between the geomagnetic field $B_{0}$ (blue) and the satellite signal $K_{i}(i=1$, 2) (red). The satellite signal $K_{i}$ (dash red line) is actually a ranging measurement of the station (green). If the satellite and its ranging measurement is conceptually regarded as a center and a radius of sphere, respectively, then the station coordinate solution can be any point on the surface of the sphere (solid red line). With more satellite signals, the station coordinate can be precisely determined. Thus, the solid red line in Fig. 5 simulates the potential positioning solution caused by the $I_{2}$ impact.

In Fig. 5, the positive value of the $B_{0} \cos \theta$ indicates that the phase measurement is longer than its geometry distance. Conversely, the negative value accounts for the phase measurement shorter than its geometry distance. For the signals from the north (azimuth angle of $0^{\circ}$ ), the phase advance is found, and the phase delay is performed with those signals from the south (azimuth angle of $180^{\circ}$ ). The intersection of two satellite signals (red) represents the positioning solution (green). This simulation leads to the station displacement moving toward the south. However, the $I_{2}$-induced ranging error ranges from a few $\mathrm{mm}$ to a few centimeters. The above two effects, namely the seasonal effect and $I_{2^{-}}$ induced error, are marginal on the SF positioning error. As such, this is not the case for the trend derived by the SF
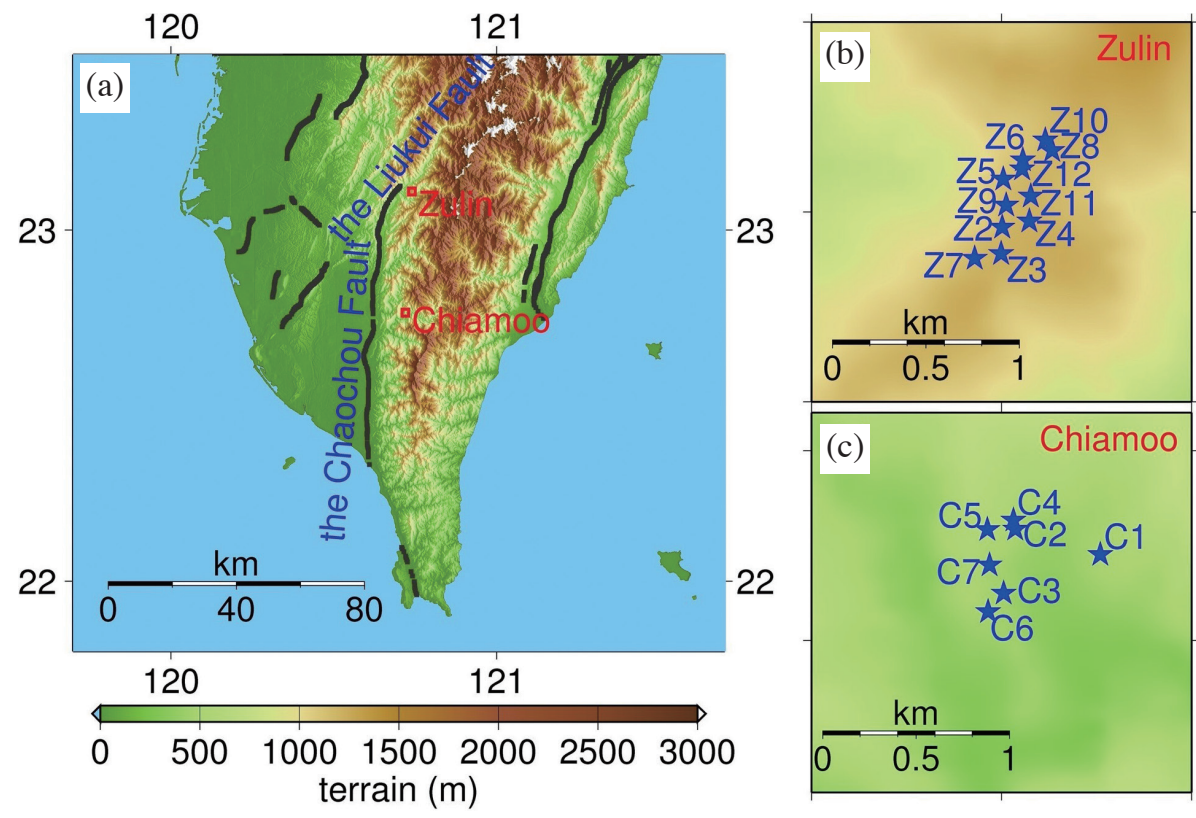

Fig. 1. (a) The locations of the study areas (Zulin and Chiamoo, southern Taiwan). The dark gray profiles mean the active faults. The distributions of the GPS stations (blue in subplots) at (b) Zulin, and (c) Chiamoo.
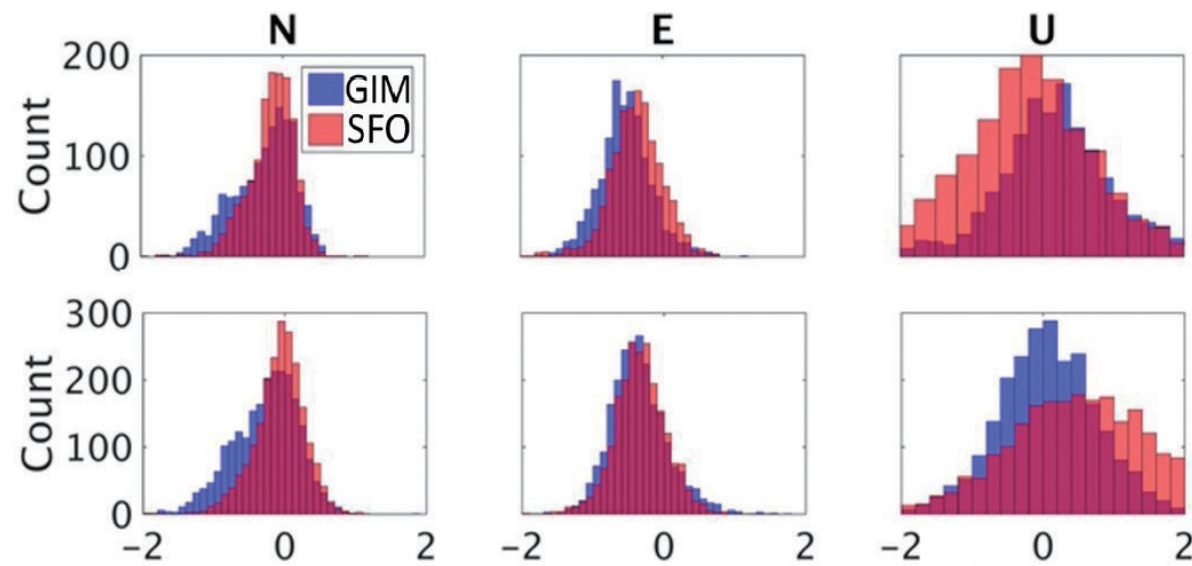

Difference $(m)$
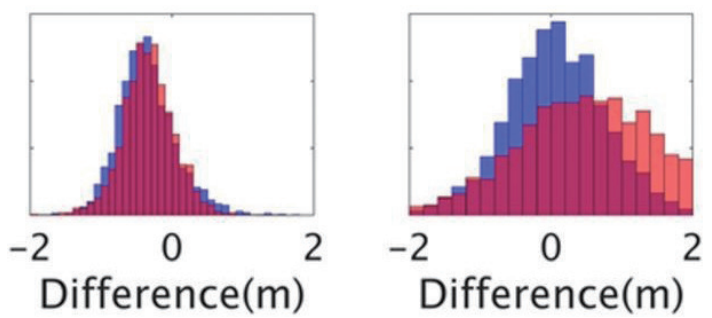

Fig. 2. Histogram of the displacements in both the GIM (blue) and the SFO (red) cases over Chiamoo (top) and Zulin (bottom). 
Table 1. Statistics of the station displacements in both the GIM and the SFO cases over Chiamoo and Zulin.

\begin{tabular}{c|cc|cc}
\hline \multirow{2}{*}{} & \multicolumn{2}{|c|}{ GIM (N/E/U) (m) } & \multicolumn{2}{c}{ SFO (N/E/U) (m) } \\
\cline { 2 - 5 } & Mean & STD & Mean & STD \\
\hline Chiamoo & $-0.26 /-0.52 / 0.18$ & $0.43 / 0.37 / 0.74$ & $-0.17 /-0.37 /-0.17$ & $0.34 / 0.37 / 0.84$ \\
Zulin & $-0.27 /-0.35 / 0.06$ & $0.46 / 0.41 / 0.68$ & $-0.08 /-0.34 / 0.41$ & $0.36 / 0.37 / 0.86$ \\
\hline
\end{tabular}
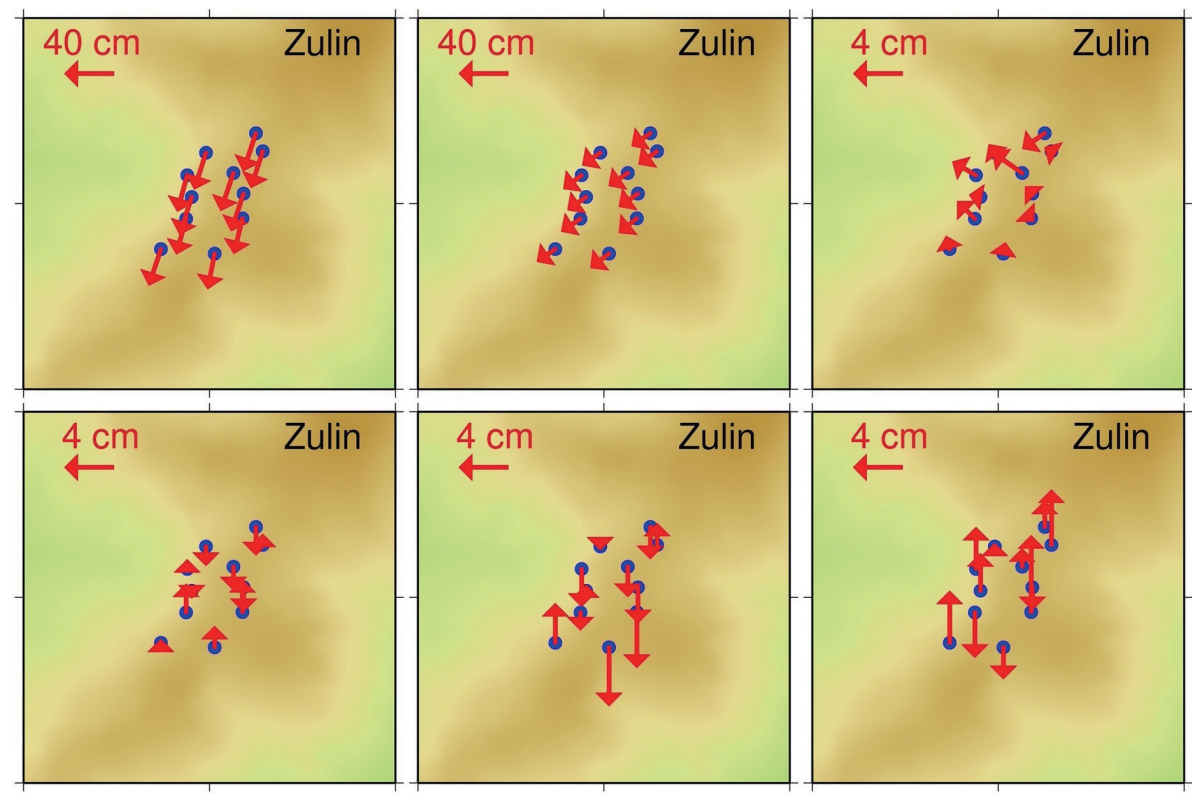

Fig. 3. Displacement trends in the horizontal (top) and vertical (bottom) components derived by GIM (left), SFO (middle) and the relative positioning (right) over Zulin. Blue dots indicate the stations in Fig. 1 and red arrows indicate the displacement trends of the stations.
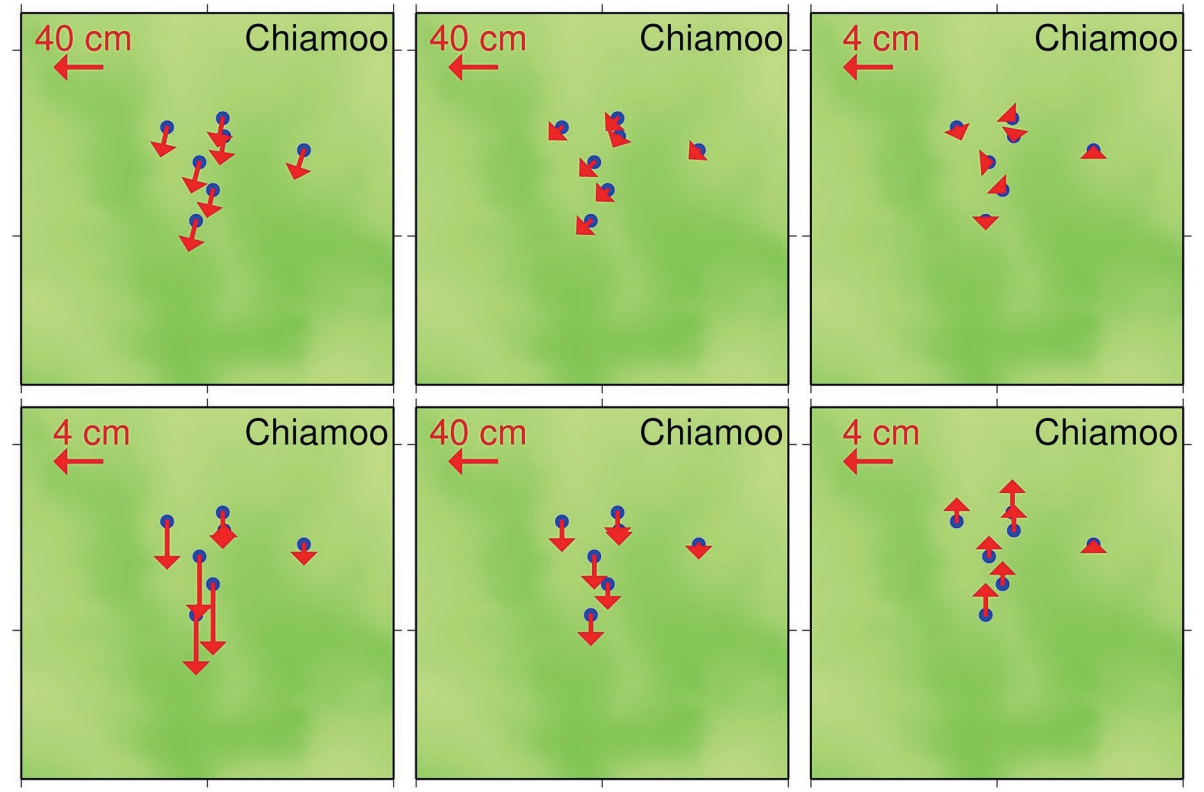

Fig. 4. Displacement trends in the horizontal (top) and vertical (bottom) components derived by GIM (left), SFO (middle) and the relative positioning (right) over Chiamoo. Blue dots indicate the stations in Fig. 1 and red arrows indicate the displacement trends of the stations. 

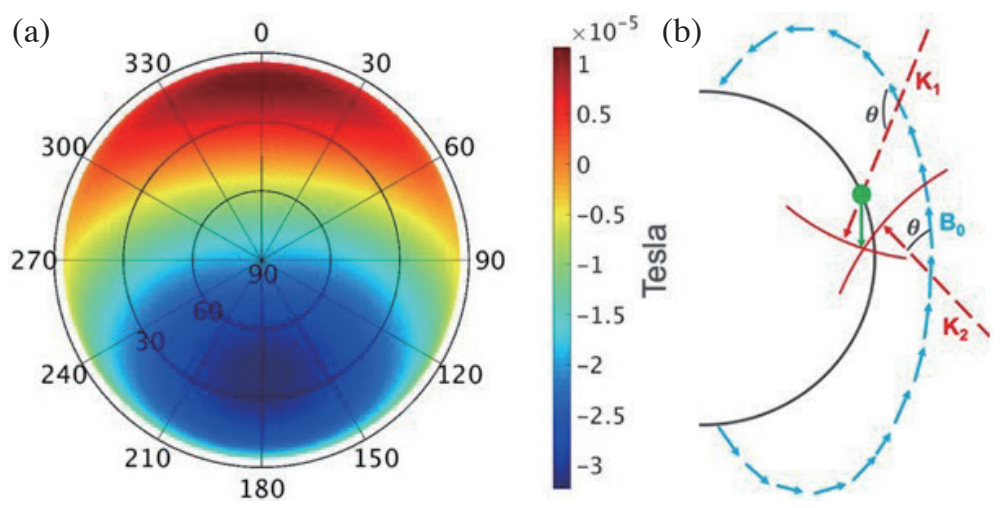

Fig. 5. Simulation of the $B_{0} \cos \theta$ (left) on a sky-plot and the illustration for the impact of the $I_{2}$ on the positioning solution, where the station (green) is located at a latitude of $22.5^{\circ}$, the geomagnetic field $B_{0}$ is in blue and the satellite signal $K_{i}(i=1,2)$ is in red.

PPP. The biased trends in Figs. 3 and 4 are most likely associated with the periodic variation of the ionospheric TEC. Note that the TEC periodic variation is here referred to the monthly and semi-annual signals (Liu et al. 2007; Guo et al. 2015), rather than the diurnal signal. In the following, both the station displacements and the daily averaged TEC variations are consistently filtered for correlation analysis. The averaged TEC is here obtained from the mean value of the sum of the daily TEC maximum and minimum over the experiment areas.

Figure 6 shows the station displacements and a short wavelength variation of the ionospheric TEC as a function of time over Zulin and Chiamoo. The short wavelength variation shows an approximate period of 27 days. Such a 27-day periodic variation is mainly caused by the solar rotation that leads to the variation of solar Extreme Ultraviolet (EUV) radiation. The solar EUV variation is mainly resulted from the non-uniform flux distribution over the solar active regions and is usually indexed by F10.7 (Smith and Gottlieb 1974; Ma et al. 2012; Vaishnav et al. 2019). Table 2 shows the maximum correlation coefficient between the TEC 27-day variation and the station displacement. The station displacement presents a consistent trend with the TEC 27-day variations. We discover a strong correlation in the $\mathrm{U}$ component, followed by the E and N components. Such a result indicates that the variation of the station displacement is associated with the TEC 27-day variation.

Figure 7 shows the station displacements and the longwavelength (semi-annual) variation, which shows the strong TECs during the equinox (March to April and September to October). We discover that the semi-annual correlation is more significant than the 27-day one. The maximum correlation coefficient is higher than 0.8 for the $\mathrm{U}$ component in both the GIM and SFO cases. This implies that the annual TEC variation directly leads to the large perturbation in the time series of the station displacements. Note that the TEC effect on the $\mathrm{N}$ component is weak in the GIM case but is significant in the SFO case. This might be due to the fact that the slant TEC is estimated in the SFO case, in which the parameters are correlated.

As a final remark, the trends of Figs. 3 and 4 results from the slope of a linear equation used to fit a time series of station displacements and the total displacement is then predicted after 217 days. The trend in the horizontal component is formed from the net of the $\mathrm{N}$ and $\mathrm{E}$ components, both of which show a negative trend so that the predicted displacement is larger than that in the vertical component. However, the correlation coefficient in Table 2 is an indicator of the consistency between the station displacement and the TEC variation. It is possible to have a low correlation but a large prediction displacement.

\section{QUANTIFICATION OF SEMI-ANNUAL TEC VARIATION EFFECT ON STATION DISPLACEMENTS}

Since the semi-annual TEC effect on the station displacement is severer than the 27-day one, we use the following model to remove the impact of the semi-annual variation on the station displacements:

$y=A \cos \left(\frac{2 \pi \cdot t}{P}-\varphi\right)$

where $y$ denotes the station displacement, $A$ denotes the amplitude, $P$ denotes the semi-annual period, $t$ denotes the data rate and $\varphi$ denotes the initial phase. In this work, the 217 days of the displacements from each station are used to estimate the amplitude and the initial phase of Eq. (3).

Table 3 shows the averaged amplitude and initial phase in the GIM and SFO cases. The impact of the semi-annual variation on the amplitude in the $\mathrm{N}, \mathrm{E}$, and $\mathrm{U}$ directions is approximately $0.71,0.42$, and $0.90 \mathrm{~m}$, respectively, for $\mathrm{Zu}$ lin and $0.71,0.48$, and $0.93 \mathrm{~m}$, respectively, for Chiamoo. This result is close to the STDs shown in Table 1, in particular for the U direction. We conclude that the SF positioning 

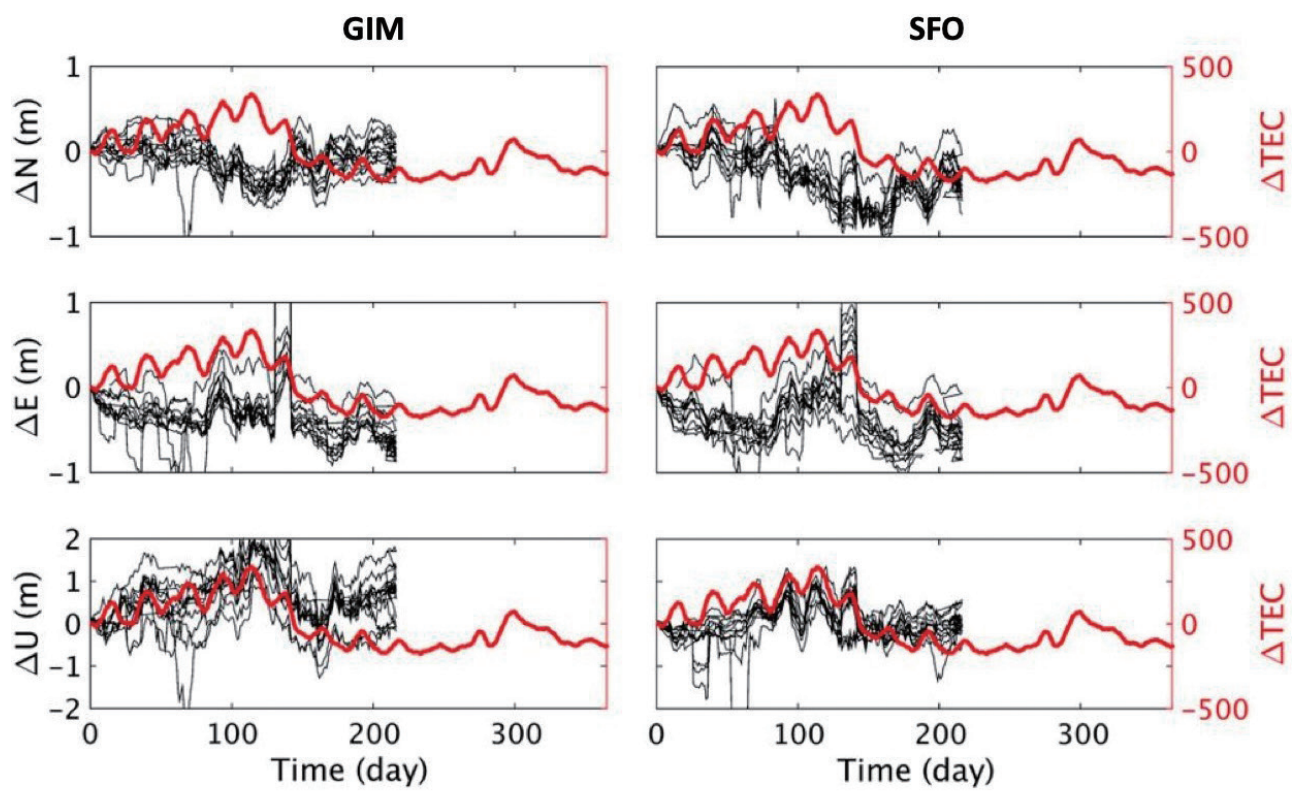

GIM

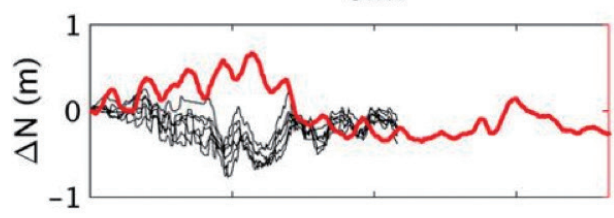

SFO
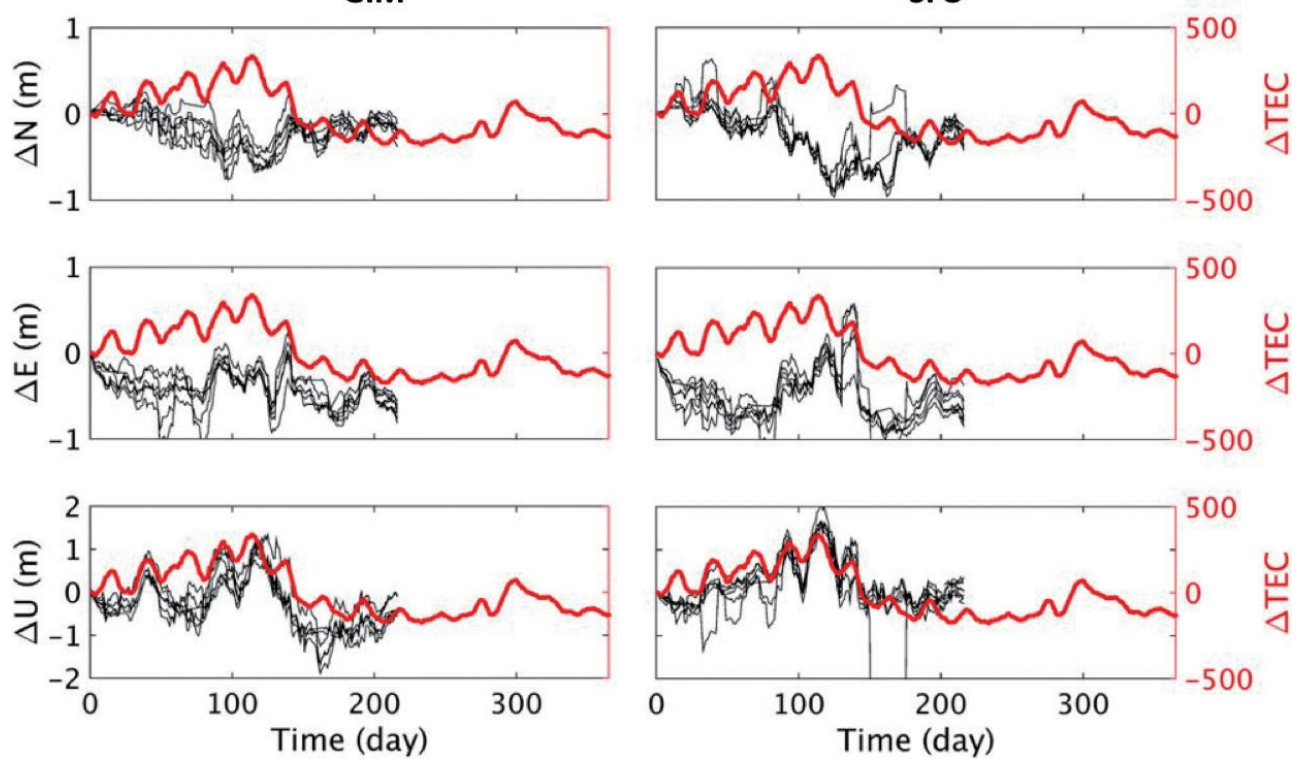

Fig. 6. Station displacements in black and the 27-day variation of the ionospheric TEC in red $\left(0.1 \times\right.$ electrons $\left./ \mathrm{m}^{2}\right)$ as a function of time over Zulin (top) and Chiamoo (bottom): GIM (left) and SFO (right).

Table 2. The maximum correlation coefficient between the TEC periodic variations and the station displacements.

\begin{tabular}{c|cc|cc}
\hline \multirow{2}{*}{} & \multicolumn{2}{|c|}{ Zulin (N/E/U) } & \multicolumn{2}{c}{ Chiamoo (N/E/U) } \\
\cline { 2 - 5 } & 27-day & Semi-annual & 27-day & Semi-annual \\
\hline GIM & $0.35 / 0.66 / 0.70$ & $-0.24 / 0.70 / 0.81$ & $-0.28 / 0.61 / 0.81$ & $-0.37 / 0.60 / 0.88$ \\
SFO & $-0.60 / 0.66 / 0.62$ & $-0.69 / 0.69 / 0.77$ & $-0.61 / 0.64 / 0.74$ & $-0.72 / 0.60 / 0.86$ \\
\hline
\end{tabular}



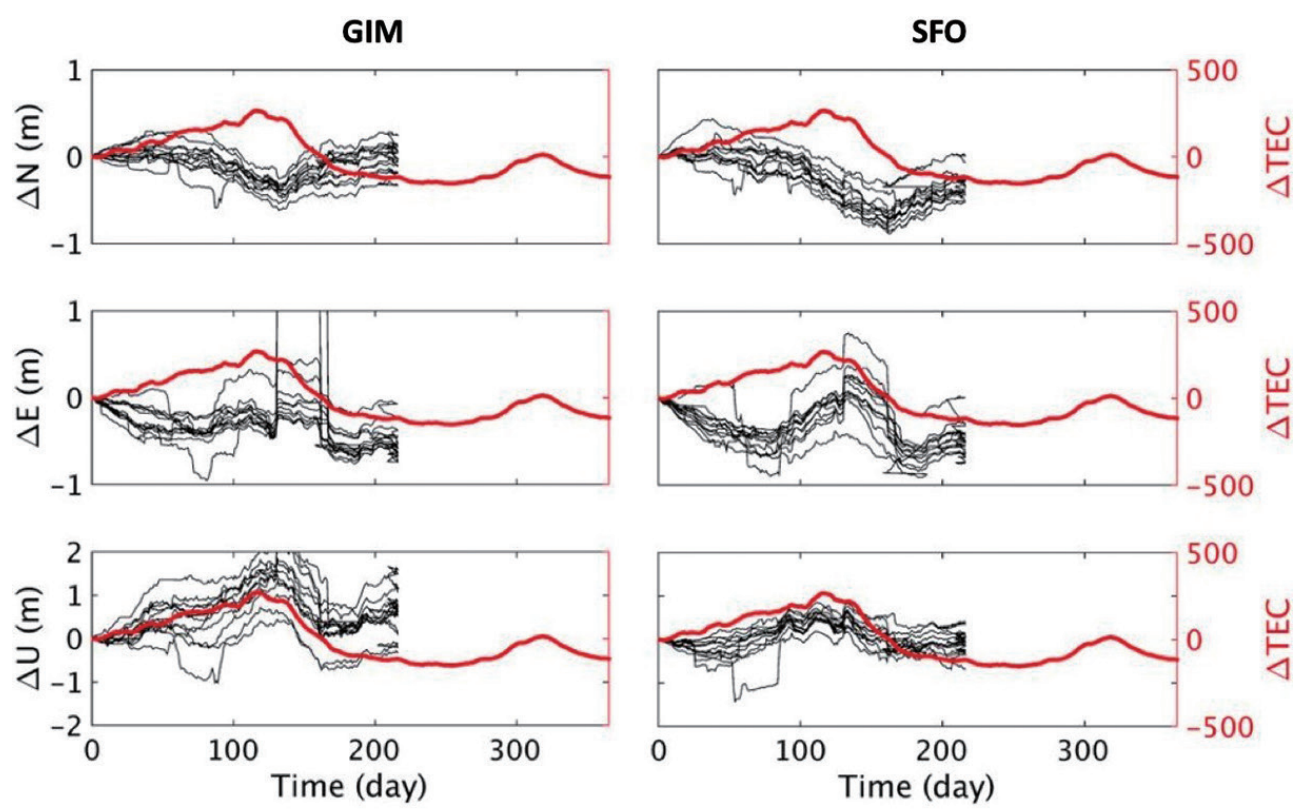

GIM

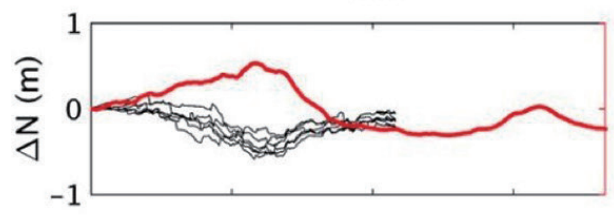

SFO
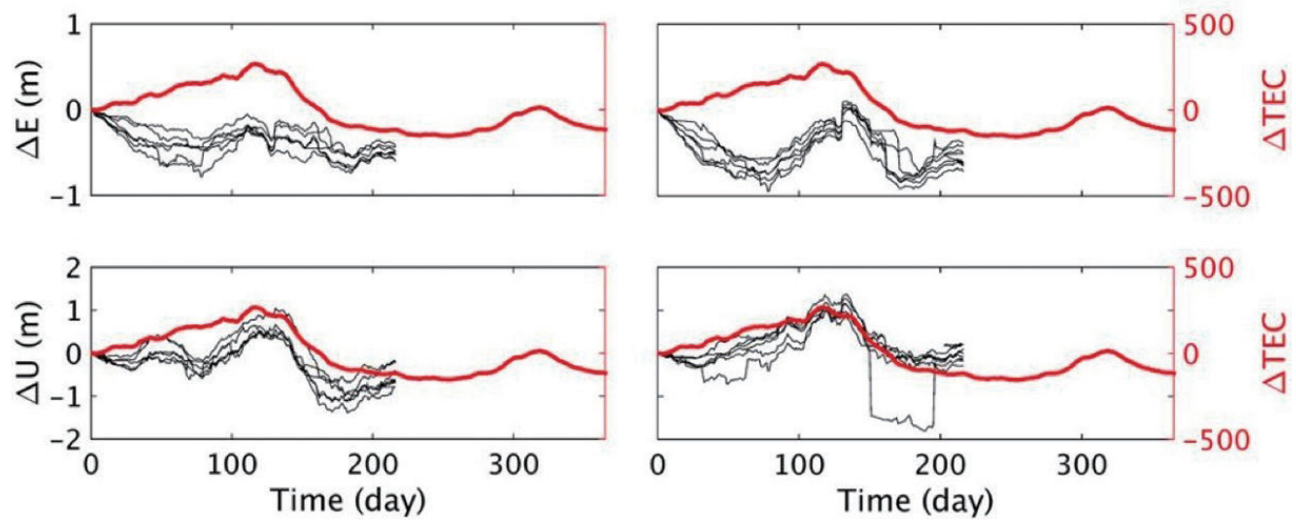

Fig. 7. Station displacements in black and the semi-annual variation of the ionospheric TEC in red $\left(0.1 \times\right.$ electrons $\left./ \mathrm{m}^{2}\right)$ as a function of time over Zulin (top) and Chiamoo (bottom): GIM (left) and SFO (right).

Table 3. Averaged amplitude and initial phase in the GIM and SFO cases.

\begin{tabular}{c|cc|cc}
\hline \multirow{2}{*}{} & \multicolumn{2}{|c|}{ Zulin (N/E/U) } & \multicolumn{2}{c}{ Chiamoo (N/E/U) } \\
\cline { 2 - 5 } & Amplitude (m) & Phase (deg) & Amplitude (m) & Phase (deg) \\
\hline GIM & $0.76 / 0.40 / 0.93$ & $111.1 / 118.3 / 209.6$ & $0.78 / 0.42 / 0.96$ & $110.1 / 112.0 / 203.0$ \\
SFO & $0.65 / 0.43 / 0.86$ & $98.5 / 105.5 / 202.1$ & $0.63 / 0.53 / 0.90$ & $95.3 / 109.3 / 199.4$ \\
\hline
\end{tabular}


error is mainly caused by the semi-annual TEC variation of the amplitude. The impact of the semi-annual variation on the phase is very similar. Only the phase in the $\mathrm{N}$ direction from the GIM is larger than that from SFO by $\sim 20$ degrees. This 20-degree phase difference might be caused by the different strategies (GIM and SFO), which are used to handle with the TEC effect on positioning.

With Table 3 information, the semi-annual TEC effect is removed from all station displacements. Figures 8 and 9 show the displacement trend without the semi-annual effect. The scale of the displacement trend is reduced to a few$\mathrm{cm}$ level, in particular for the horizontal component. Additionally, the displacement trend of the vertical component is consistent with those given by the relative positioning method. This strongly suggests that the semi-annual TEC effect should be removed from the time series of the station displacements derived by the SF PPP. Although a systematic moment is still found in the SFO case of Figs. 8 and 9, the scale of the system is relatively small as compared to that in Figs. 3 and 4. We suspect that such a small system movement might be caused by the annual TEC variation, whose wavelength is longer than the semi-annual one. This needs to be further investigated.

If both the amplitude and the phase of Eq. (3) can be precisely estimated and then be corrected to the station displacement, the SF-derived positioning solution may be improved. That is $x_{i}=x_{i+1}-A \cos \left(\frac{2 \pi \cdot t_{i}}{P}-\varphi\right)$, where $x_{i}$ denotes the SF-derived station position on the $i$-th day and the $x_{i+1}$ denotes the station position on the next day of $x_{i}$.

\section{CONCLUSIONS}

In this study, we discover the strong correlation between the semi-annual TEC variation and the station displacements derived by the SF PPP technique, in which the ionospheric delay is mitigated using the GIM and SFO strategies for the internal comparison. In both cases, the displacement trend performs the systematic movement toward the southwest (Figs. 3 and 4) and is very sensitive to the semi-annual TEC variations (Table 2).

The maximum correlation coefficient is higher than 0.8 in the station $\mathrm{U}$ component, followed by the $\mathrm{E}$ and $\mathrm{N}$ components. Furthermore, we also quantify the semi-annual TEC effect on station displacement. The impact of the semi-annual TEC variation on station displacement in the $\mathrm{N}, \mathrm{E}$, and $\mathrm{U}$ directions is approximately $0.71,0.42$, and $0.90 \mathrm{~m}$, respectively, for Zulin and $0.71,0.48$, and $0.93 \mathrm{~m}$, respectively, for Chiamoo. Such an impact is geographically dependent and is close to the STDs shown in Table 1, in particular for the $\mathrm{U}$ direction. The SF positioning error is sensitive to the semiannual TEC variation. This suggests that the semi-annual effect should be removed from the time series of the SFderived station displacements. In other words, if both the amplitude and the phase of Eq. (3) are precisely estimated and are then corrected to the station displacement, both the SF-derived positioning accuracy and the displacement trend may be improved. By doing so, the overall cost for the deployment of the station networks can be greatly reduced. This study may serve as a reference for assessing the impact of the semi-annual TEC variation on the time series of the station displacement using the SF PPP technique.

Acknowledgements This project is supported by the Ministry of Science and Technology (MOST) of Taiwan under Grants 104-2625-M-008-004, MOST 105-2625-M-008011, 102-2116-M-008-001, and 105-2221-E-005-014. We are grateful to the Central Geological Survey of Taiwan who provided the GPS data over Zulin and Chiamoo for this study. We acknowledge CODE for providing the precise GPS orbit, clock and earth rotation parameters.

\section{REFERENCES}

Blewitt, G. and D. Lavallee, 2002: Effect of annual signals on geodetic velocity. J. Geophys. Res., 107, ETG 9-1ETG 9-11, doi: 10.1029/2001JB000570. [Link]

Boehm, J., A. Niell, P. Tregoning, and H. Schuh, 2006: Global Mapping Function (GMF): A new empirical mapping function based on numerical weather model data. Geophys. Res. Lett., 33, L07304, doi: 10.1029/2005GL025546. [Link]

Dach, R., S. Lutz, P. Walser, and P. Fridez, 2015: Bernese GNSS Software Version 5.2, University of Bern, Bern Open Publishing, Bern, Switzerland, 852 pp, doi: 10.7892/boris.72297. [Link]

Gao, Y. and Z. Z. Liu, 2002: Precise ionosphere modeling using regional GPS network data. J. Glob. Position. Syst., 1, 18-24.

Guo, J., W. Li, X. Liu, Q. Kong, C. Zhao, and B. Guo, 2015: Temporal-spatial variation of global GPS-derived total electron content, 1999-2013. PLOS ONE, 10, e0133378, doi: 10.1371/journal.pone.0133378. [Link]

Hadas, T., A. Krypiak-Gregorczyk, M. Hernández-Pajares, J. Kaplon, J. Paziewski, P. Wielgosz, A. Garcia-Rigo, K. Kazmierski, K. Sosnica, D. Kwasniak, J. Sierny, J. Bosy, M. Pucilowski, R. Szyszko, K. Portasiak, G. Olivares-Pulido, T. Gulyaeva, and R. Orus-Perez, 2017: Impact and implementation of higher-order ionospheric effects on precise GNSS applications. J. Geophys. Res., 122, 9420-9436, doi: 10.1002/2017JB014750. [Link]

Hastaoglu, K. O. and D. U. Sanli, 2011: Monitoring Koyulhisar landslide using rapid static GPS: A strategy to remove biases from vertical velocities. Nat. Hazards, $\mathbf{5 8}$, 1275-1294, doi: 10.1007/s11069-011-9728-5. [Link]

Hernández-Pajares, M., J. M. Juan, J. Sanz, and R. Orús, 

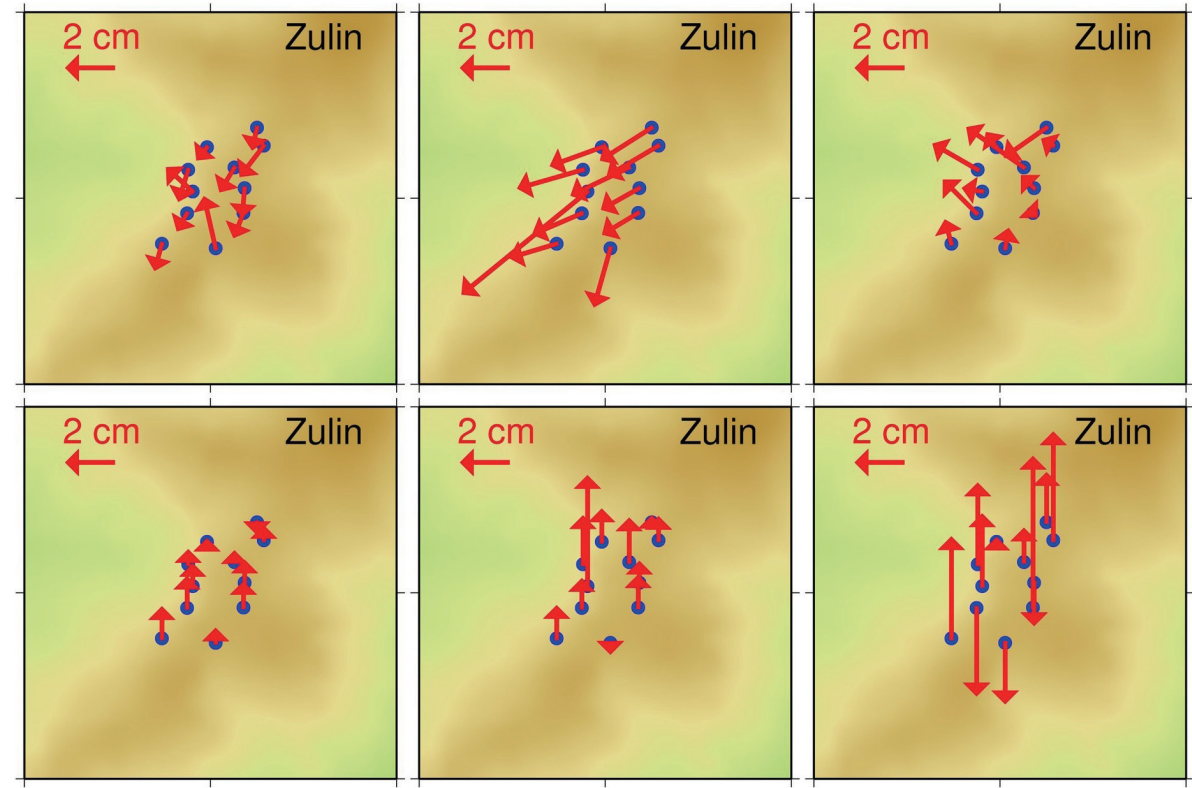

Fig. 8. Displacement trends without the semi-annual TEC effect in the horizontal (top) and vertical (bottom) components derived by GIM (left), SFO (middle) and the relative positioning (right) over Zulin. Blue dots indicate the stations in Fig. 1 and red arrows indicate the displacement trends of the stations.
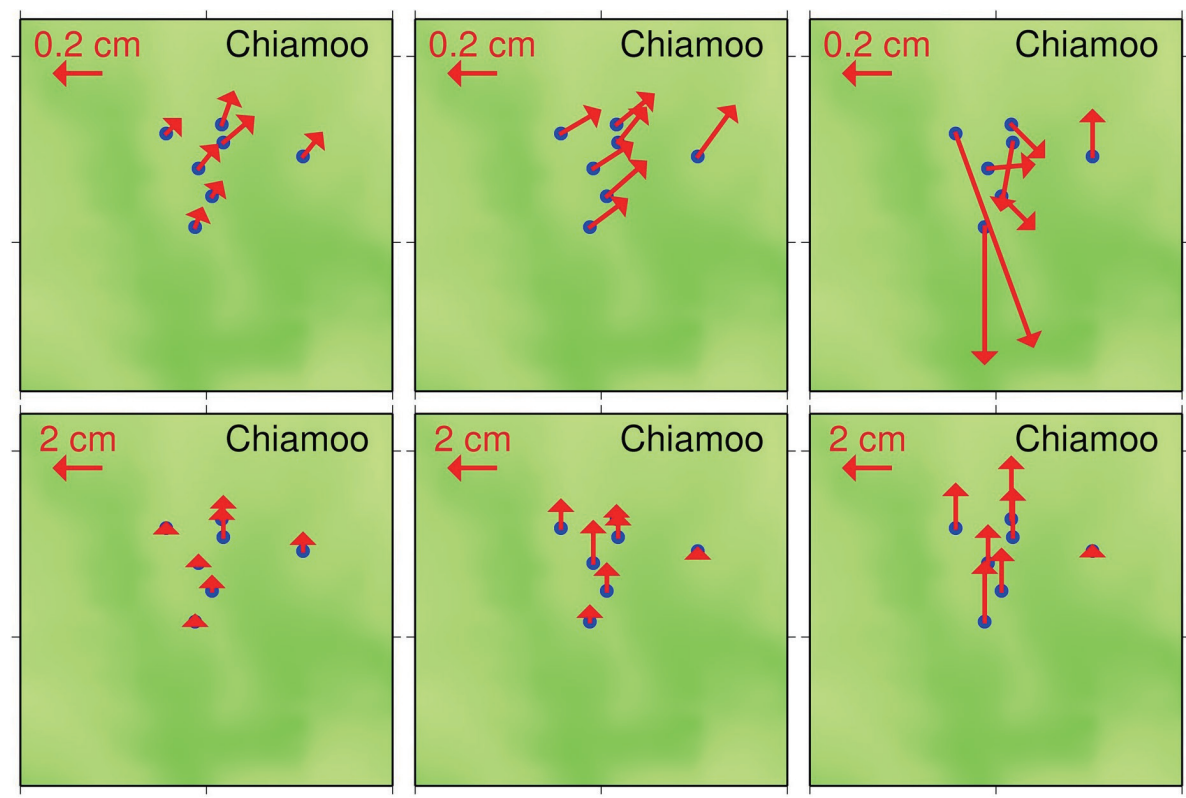

Fig. 9. Displacement trends without the semi-annual TEC effect in the horizontal (top) and vertical (bottom) components derived by GIM (left), SFO (middle) and the relative positioning (right) over Chiamoo. Blue dots indicate the stations in Fig. 1 and red arrows indicate the displacement trends of the stations. 
2007: Second-order ionospheric term in GPS: Implementation and impact on geodetic estimates. J. Geophys. Res., 112, B08417, doi: 10.1029/2006JB004707. [Link]

Hofmann-Wellenhof, B., H. Lichtenegger, and J. Collins, 2001: Global Positioning System: Theory and Practice, Springer-Verlag Wien, New York, 382 pp, doi: 10.1007/978-3-7091-6199-9. [Link]

Jin, S., J.-U. Park, J.-H. Cho, and P.-H. Park, 2007: Seasonal variability of GPS-derived zenith tropospheric delay (1994-2006) and climate implications. J. Geophys. Res., 112, D09110, doi: 10.1029/2006JD007772. [Link]

Kedar, S., G. A. Hajj, B. D. Wilson, and M. B. Heflin, 2003: The effect of the second order GPS ionospheric correction on receiver positions. Geophys. Res. Lett., 30, doi: 10.1029/2003GL017639. [Link]

Liu, A., N. Wang, Z. Li, K. Zhou, and H. Yuan, 2018: Validation of CAS's final global ionospheric maps during different geomagnetic activities from 2015 to 2017. Results Phys., 10, 481-486, doi: 10.1016/j. rinp.2018.06.057. [Link]

Liu, L., B. Zhao, W. Wan, S. Venkartraman, M.-L. Zhang, and X. Yue, 2007: Yearly variations of global plasma densities in the topside ionosphere at middle and low latitudes. J. Geophys. Res., 112, A07303, doi: 10.1029/2007JA012283. [Link]

Ma, R., J. Xu, W. Wang, and J. Lei, 2012: The effect of $\sim 27$ day solar rotation on ionospheric $F_{2}$ region peak densities $\left(N_{m} F_{2}\right)$. J. Geophys. Res., 117, A03303, doi: 10.1029/2011JA017190. [Link]
Meier, U., N. M. Shapiro, and F. Brenguier, 2010: Detecting seasonal variations in seismic velocities within Los Angeles basin from correlations of ambient seismic noise. Geophys. J. Int., 181, 985-996, doi: 10.1111/j.1365246X.2010.04550.x. [Link]

Smith, E. V. P. and D. M. Gottlieb, 1974: Solar flux and its variations. Space Sci.Rev., 16, 771-802, doi: 10.1007/ BF00182600. [Link]

Tseng, T.-P., C. Hwang, K. Sośnica, C.-Y. Kuo, Y.-C. Liu, and W.-H. Yeh, 2017: Geocenter motion estimated from GRACE orbits: The impact of F10.7 solar flux. Adv. Space Res., 59, 2819-2830, doi: 10.1016/j. asr.2016.02.003. [Link]

Tseng, T.-P., S.-Y. Chen, K.-L. Chen, C.-Y. Huang, and W.-H. Yeh, 2018: Determination of near real-time GNSS satellite clocks for the FORMOSAT-7/COSMIC-2 satellite mission. GPS Solut., 22, doi: 10.1007/ s10291-018-0714-1. [Link]

Vaishnav, R., C. Jacobi, and J. Berdermann, 2019: Longterm trends in the ionospheric response to solar extreme-ultraviolet variations. Ann. Geophys., 37, 11411159, doi: 10.5194/angeo-37-1141-2019. [Link]

Wang, G.-Q., 2012: Kinematics of the Cerca del Cielo, Puerto Rico landslide derived from GPS observations. Landslides, 9, 117-130, doi: 10.1007/s10346-0110277-5. [Link]

Zumberge, J. F., M. B. Heflin, D. C. Jefferson, M. M. Watkins, and F. H. Webb, 1997: Precise Point Positioning for the efficient and robust analysis of GPS data from large networks. J. Geophys. Res., 102, 5005-5017, doi: 10.1029/96JB03860. [Link] 